\title{
Experimental Study of Turbulence and Zonal Flow in Edge Plasmas of the HL-2A Tokamak
}

\author{
Jiaqi DONG ${ }^{1,4)}$, Kaijun ZHAO ${ }^{1)}$, Longwen YAN ${ }^{1)}$, Wenyu HONG ${ }^{1)}$, Changxuan $\mathrm{YU}^{2)}$, \\ Akihide FUJISAWA ${ }^{3)}$, Jun QIAN ${ }^{1)}$, Jun CHENG $^{1)}$, Adi LIU ${ }^{2)}$, Tao LAN $^{2)}$, Hailin ZHAO ${ }^{2)}$, \\ Defeng $\mathrm{KONG}^{2)}$, Yi $\mathrm{LIU}^{1)}$, Yuan $\mathrm{HUANG}^{1)}$, Qiang $\mathrm{LI}^{1)}$, Xianming $\mathrm{SONG}^{1)}$, Qingwei YANG ${ }^{1)}$, \\ Xuantong DING ${ }^{1)}$, Xuru DUAN ${ }^{1)}$ and Yong LIU ${ }^{1)}$ \\ ${ }^{1)}$ Southwestern Institute of Physics, P. O. Box 432, Chengdu, China \\ ${ }^{2)}$ Department of Modern Physics, University of Science and Technology of China, Hefei, China \\ ${ }^{3)}$ National Institute for Fusion Science, 322-6 Oroshi-cho, Toki 509-5292, Japan \\ ${ }^{4)}$ Institute for Fusion Theory and Simulation, Zhejiang University, Hangzhou, China
}

(Received 7 December 2009 / Accepted 4 March 2010)

\begin{abstract}
Measurements with a three dimensional set of Langmuir probe arrays have unambiguously demonstrated the coexistence of intensive low frequency zonal flows (LFZFs), geodesic acoustic modes (GAMs), low frequency fluctuations (LFFs) and high frequency ambient turbulence (HFAT) in the edge of HL-2A tokamak plasmas, by verifying the temporal and spatial characteristics of the electrostatic fluctuations. The intensity of the LFZFs is observed to increase and decrease with increases of ECRH power $(\sim 300-700 \mathrm{~kW})$ and safety factor $q(\sim 3.5$ 6.2 ), respectively, and the intensity of the GAMs increases with the ECRH power as well as $q$. The radial wave number-frequency spectra of the LFZF show that the LFZF packets propagate outwards and inwards as equally likely events, while the GAM packets propagate predominantly outwards. The three wave coupling of the zonal flows, including the GAMs, and the LFFs with the HFAT is investigated in detail.
\end{abstract}

(C) 2010 The Japan Society of Plasma Science and Nuclear Fusion Research

Keywords: turbulence, zonal flow, geodesic acoustic mode, coherency, Langmuir probe

DOI: $10.1585 /$ pfr.5.S2014

\section{Introduction}

The anomalous cross field transport in magnetically confined plasmas is generally attributed to microfluctuations called turbulence. Formation of meso- and large-scale structures such as zonal flows (ZFs) is universal in such turbulent systems. The ZFs in magnetically confined plasmas are defined as azimuthally symmetric radial electric field fluctuations with finite radial wavelengths. It is widely accepted in recent decades that the turbulence and induced turbulent transport may be reduced or even suppressed by zonal flows [1] and sheared mean flows [2,3]. Therefore, extensive studies have been carried out to understand the physics mechanisms responsible for the generation of the flows and the resulting reduction of the transport induced by turbulent fluctuations. Two types of zonal flows have been studied in magnetically confined toroidal plasmas, i.e., near zero low frequency zonal flows (LFZFs) [4,5], and oscillatory flows termed geodesic acoustic modes (GAMs) [6]. The GAMs were first identified in experiment on DIII-D device [7] and have been extensively studied in recent years [8]. For example, the toroidal symmetry of the GAMs was first measured on HL2A tokamak [9] while the fluctuation-driven particle flux was found to be modulated at the frequency of the observed GAM oscillations on JFT-2 M device [10]. The suppression of turbulent fluctuation through LFZF was also observed in CHS plasmas using two heavy-ion beam probes [11]. A low-frequency broadband (from zero to $2 \mathrm{kHz}$ ) feature in the potential fluctuations was directly measured recently in the edge region of HL-2A plasmas [12]. The aim of the above mentioned studies is to explore physics mechanisms leading to ZF formation, turbulent transport and its reduction. The investigation is still at an initial stage and much work is desirable. For example, dynamics of ATs in the presence of GAMs or LFZFs has not been reported in tokamak experiments. The spectrum structures of the turbulence have not been reported in detail. In addition, physics mechanisms for AT-ZF interaction have not been revealed thoroughly.

This work unambiguously demonstrate that intensive low frequency zonal flows (LFZFs), geodesic acoustic modes (GAMs), low frequency fluctuations (LFFs) and high frequency ambient turbulence (HFAT) coexist in the edge of HL-2A tokamak plasmas. The tempo-spatial features of the flows are analyzed in detail. In addition, nonlinear three wave coupling of LFZF, GAM, and LFFs with the HFAT is also investigated. 


\section{Experiment Arrangement}

The experiments presented here were conducted in Ohmic- and ECRH-heated deuterium plasma of the HL$2 \mathrm{~A}$ tokamak with major and minor radii $R=1.65 \mathrm{~m}$ and $a=0.4 \mathrm{~m}$, respectively. The parameters used are: toroidal magnetic field $B_{\mathrm{t}}=1.2 \mathrm{~T}$, plasma current $I_{\mathrm{p}}=110-150$ $\mathrm{kA}$, line-averaged electron density $\bar{n}_{\mathrm{e}}=1-2 \times 10^{19} \mathrm{~m}^{-3}$, boundary safety factor $q_{a}=3.5-6.2$, discharge duration $t_{\mathrm{d}}=1.2 \mathrm{~s}$. The ion Larmour radius was estimated to be $0.2-1 \mathrm{~mm}$. The collision frequency and the safety factor at the LP locations were estimated to be $v_{\mathrm{ii}} \sim 1-7 \times 10^{3} \mathrm{~s}^{-1}$ and $q(r / a=0.92)=0.88 q_{a}$, respectively.

The floating-potential fluctuations were measured using a poloidally-oriented 10-tip and a radially-oriented 12 tip rake Langmuir probe (LP) array, both of $4 \mathrm{~mm}$ tip separation. A three-step LP array of 6 tips [9] and the radial LP array form a fast reciprocating probe set with 18 tips and a $74 \mathrm{~mm}$ poloidal span. The set, in the toroidal direction, is located in a toroidal cross section $2100 \mathrm{~mm}$ away from the poloidal LP array. The tip size and the mount of the LP sets are the same as in Ref. [9]. Unless otherwise stated, the probe data sampling rate is $1 \mathrm{MHz}$ and the frequency resolution is $0.5 \mathrm{kHz}$ in the analysis.

\section{Experimental Results}

Figure 1 shows a representative auto-power spectrum of the floating potential at an inner flux surface (IFS) of $28 \mathrm{~mm}$ inwards from the last closed flux surface (LCFS). Two distinct features are a large power fraction in the frequency range lower than $\sim 4 \mathrm{kHz}$ and a sharp peak at $f \sim 17 \mathrm{kHz}$. The former will be a priori called LFZF, and the latter has already been identified as a GAM $[9,13,14]$. In this case, the spectral power fraction for the LFZF, the GAM, and the turbulence are roughly 0.10:0.09:0.81. It is worthwhile to note that the turbulence here includes two regimes: the LFFs of $20 \mathrm{kHz}<f<100 \mathrm{kHz}$ and mesospatial scales, and the HFAT of $100 \mathrm{kHz}<f<500 \mathrm{kHz}$ and small spatial scales [15].

The coherency between the floating potentials is examined in order to verify the poloidal symmetry of the features. Figures 2 (a) and 2 (b) show the coherency and the corresponding phase shift for poloidal separations of $4 \mathrm{~mm}$ and $36 \mathrm{~mm}$ (the red and green lines), respectively, at the IFS. The phase shift is almost zero and the coherency of $\sim 0.9$ is quite high in the LFZF and GAM frequency regions. Averaged over the half width of the spectrum region, the poloidal mode number is estimated to be $m \sim 0.31 \pm 0.06$ for the LFZF, similar to the results of $m \sim 0.45 \pm 0.07$ for the GAM. It is clear that the differences between the observations with separations of $4 \mathrm{~mm}$ and $36 \mathrm{~mm}$ are negligible in the LFZF and GAM frequency regions, indicating that contributions from short poloidal wavelength fluctuations are negligibly small in these frequency regions. However, significant difference between the coherency does exhibit in the LFF regime where the co-

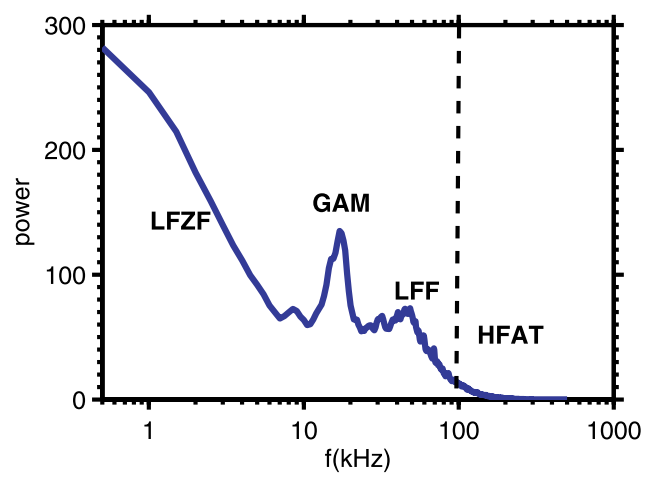

Fig. 1 A representative auto-power spectrum of the floating potential.

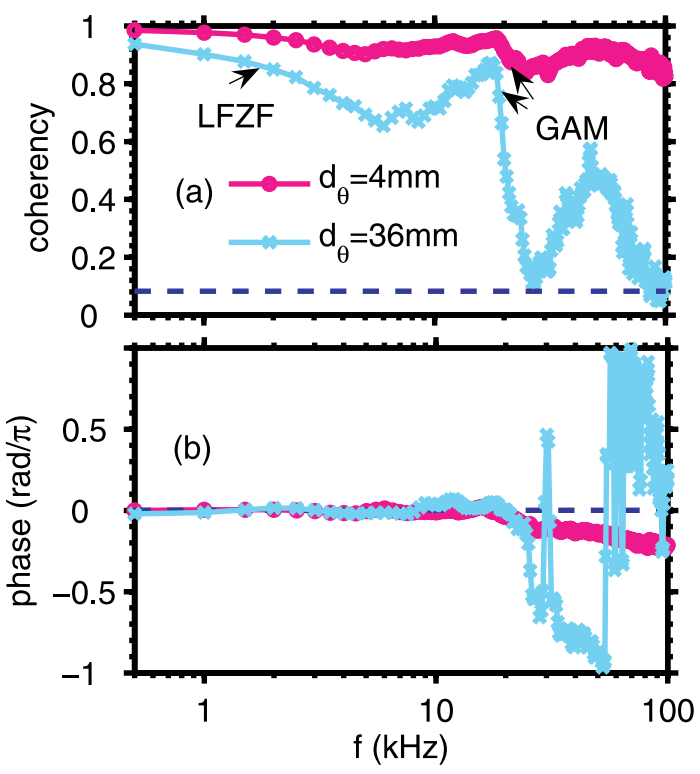

Fig. 2 (a) The coherency spectra with poloidal span of 4 and $36 \mathrm{~mm}$, and (b) the corresponding phase shift spectra.

herency with $4 \mathrm{~mm}$ separation is much higher than that of $36 \mathrm{~mm}$ separation. This means that the contribution from fluctuations of short poloidal wavelength is notable in this frequency regime. On the other hand, the coherency of $\sim 0.5$ in the LFF regime for $36 \mathrm{~mm}$ separation is still relatively high, indicating rather strong correlation over such a poloidal separation in this regime, in significant contrast with that in the HFAT regime where the coherency over the $36 \mathrm{~mm}$ separation drops to noise level but keeps rather high over the $4 \mathrm{~mm}$ separation. The phase shift spectra show approximately linear dispersion relations in the LFF regime for both separations but with significant difference in the slopes. The slope of the $36 \mathrm{~mm}$ separation is higher than that of the $4 \mathrm{~mm}$ separation. This means that the phase velocity of the components with longer poloidal wavelengths is higher than that of shorter poloidal wavelengths. Figures 3 (a) and 3 (b) show the coherency and the phase shift spectra for toroidal separation of $2100 \mathrm{~mm}$ at the IFS. The the coherency of $\sim 0.8$ is quite high and phase shift is also close to zero in the LFZF and GAM fre- 


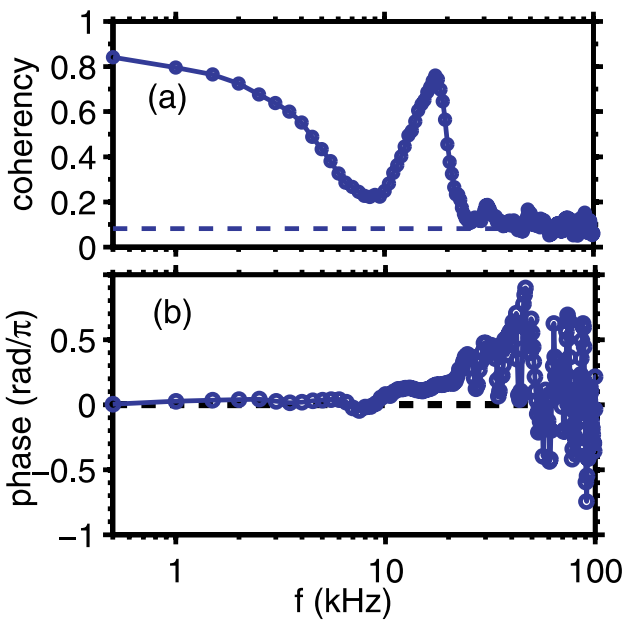

Fig. 3 (a) The toroidal coherency spectrum, and (b) corresponding phase shift spectrum.

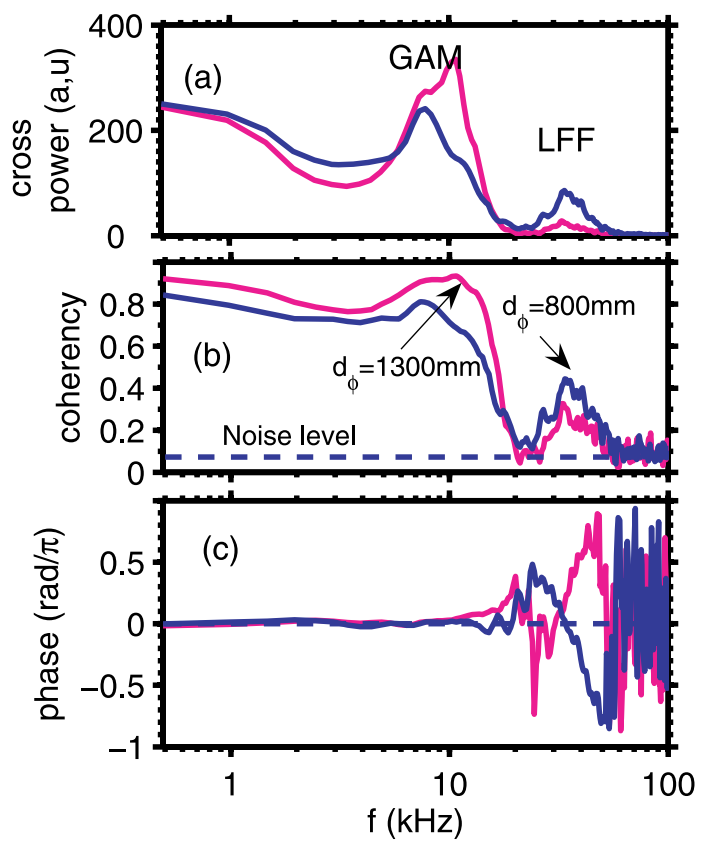

Fig. 4 (a) The toroidal cross-power spectra, (b) coherency spectra, (c) cross phase spectra of the floating potentials over toroidal spans of 800 and $1300 \mathrm{~mm}$.

quency regions. Averaged over the half width of the spectrum region, the toroidal mode number is estimated to be $n \sim 0.020 \pm 0.004$ for the LFZF, similar to the results of $m \sim 0.33 \pm 0.05$ for the GAM. The coherency drops to the noise level and the phase shift is random in the LFF regime. This may indicate that the correlation length of the LFFs in this direction is much shorter than the distance between the two probe arrays. The coherency is not significantly low and the phase shift exhibits clear linear dispersion relations in the LFF regime when the toroidal separation is $800 \mathrm{~mm}$ or even $1300 \mathrm{~mm}$ at IFS as shown in Fig. 4. In addition, the coherency for the $800 \mathrm{~mm}$ separation is lower than that for $1300 \mathrm{~mm}$, indicating the decrease of the coherency with toroidal separation and consistent with the results in Fig. 3.
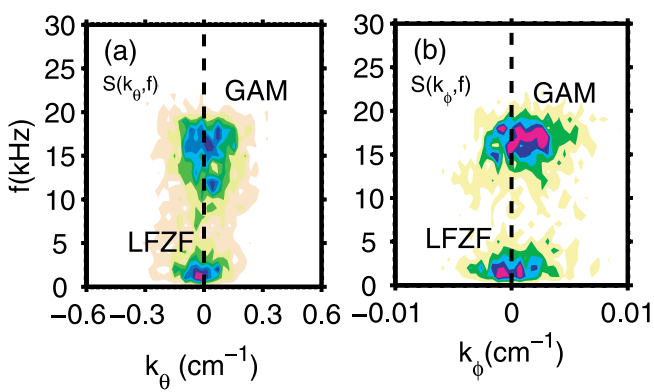

Fig. 5 (a) $S\left(k_{\theta}, f\right)$, and (b) $S\left(k_{\phi}, f\right)$ of the floating potential fluctuations.
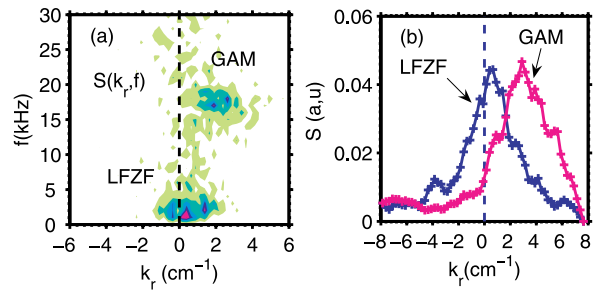

Fig. 6 (a) Radial wavenumber-frequency spectrum, $S\left(k_{r}, f\right)$ of the floating potentials between the IFS and OFS, (b) The radial wavenumber spectra of the floating potential for the LFZF and GAM.

The poloidal and toroidal wavenumber-frequency spectra, $S\left(k_{\theta}, f\right)$ and $S\left(k_{\phi}, f\right)$, of the floating potential are obtained using the two-point correlation technique [13] and shown in Figs. 5 (a) and (b). Again, it is clearly shown that the LFZF and GAM are concentrated in the frequency regions of $0<f<5 \mathrm{kHz}$ and $12 \mathrm{kHz}<f<20 \mathrm{kHz}$, respectively, and both have $k_{\phi} \sim k_{\theta} \sim 0$.

The radial wavenumber-frequency spectrum, $S\left(k_{r}, f\right)$, of the floating potential between the IFS and an outer flux surface (OFS) of $24 \mathrm{~mm}$ inward from the LCFS is shown in Fig. 6(a). The fluctuation power of the LFZF and GAM are concentrated in the radial wavenumber spectra of $-1.5 \mathrm{~cm}^{-1}<k_{r}<2.0 \mathrm{~cm}^{-1}$, and $1.0 \mathrm{~cm}^{-1}<k_{r}<$ $3.0 \mathrm{~cm}^{-1}$, respectively. The radial wavenumber spectra $S\left(k_{r}\right)$ at the averaged LFZF and GAM frequencies are presented in Fig. 6 (b). The spectrum averaged wavenumbers and half-widths are estimated to be $k_{r}=0.5 \mathrm{~cm}^{-1}$ and $\Delta k_{r}=3.7 \mathrm{~cm}^{-1}$, and $k_{r}=3.0 \mathrm{~cm}^{-1}$ and $\Delta k_{r}=4.0 \mathrm{~cm}^{-1}$ for the LFZF and GAM, respectively. In addition, it is clearly shown that the LFZF packets propagate outwards and inwards as equally likely events, while the GAM packets propagate predominantly outwards.

An essential characteristic of the ZFs is their interaction with the AT through nonlinear three-wave coupling. The bicoherency analysis, an indicator for the strength of such coupling [16], is employed to monitor the intensity of the interaction. Figures 7 (a) and (b) show the autobicoherency contour diagram for the floating potential and the part near the horizontal axis, zoomed in. The frequency resolution here is $1 \mathrm{kHz}$, so that a sufficient number of realizations can be obtained. In the figures, the fre- 


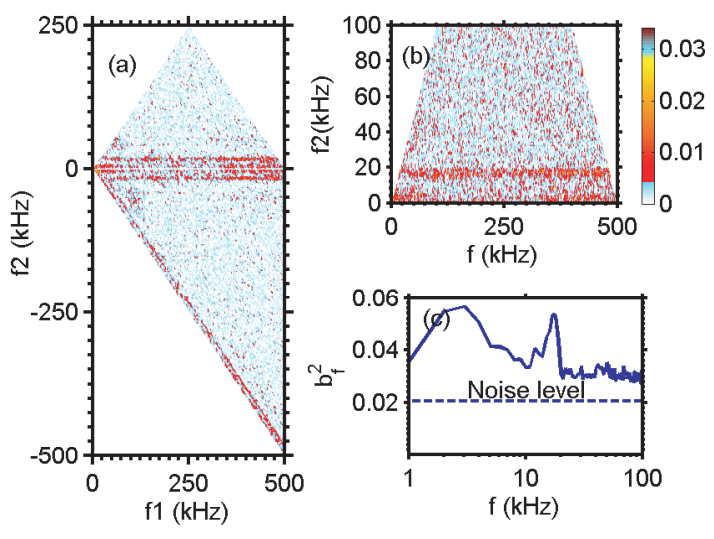

Fig. 7 (a)and (b) Squared auto-bicoherncy, (c) the summed squared auto-bicoherency.

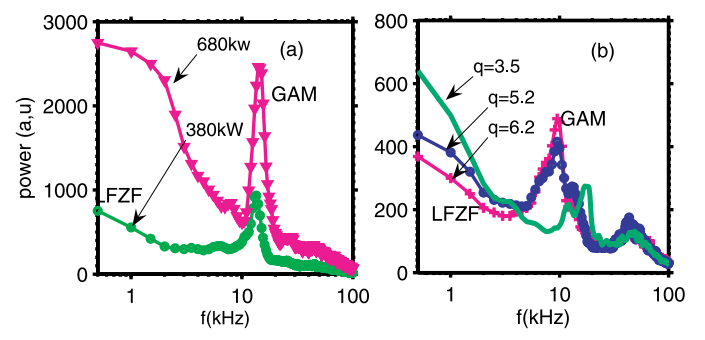

Fig. 8 Auto-power spectra of floating potentials (a) ECRH power, and (b) $q$ scanning.

quency $f_{3}$ of the third wave is determined from the matching condition $f_{1}+f_{2}=f_{3}$, where $f_{1}$ and $f_{2}$ are the frequencies of the first and second waves, respectively. The color at each point of $\left(f_{1}, f_{2}\right)$ illustrates the strength of the coupling with the third wave. The bicoherency values around $f_{3}=f_{1}-\left|f_{2}\right|<4 \mathrm{kHz}$ and $f_{2}<4 \mathrm{kHz}$, as well as $f_{3}=f_{1}-\left|f_{2}\right| \sim 17 \mathrm{kHz}$ and $f_{2} \sim 17 \mathrm{kHz}$, are much higher than the rest and significantly above the noise level. This shows that strong three-wave coupling takes place between the LFZF/GAM and the AT. The summed auto-bicoherency given in Figure 7 (c) also shows clear peaks in the LFZF and GAM frequency regions. It can thus be concluded that besides the azimuthal symmetries and radial meso-scaling, the observed LFZFs and GAM also have high power fraction and participate in strong nonlinear three-wave interaction with the AT. There is also strong three wave coupling between the LFFs and the HFAT [15].

Dependence of the ZF intensity on the plasma parameters and discharge conditions is important to theories for improvement in tokamak plasma confinement, such as transport barrier formation, low-to-high confinement transition, etc. The spectra of the ZF were examined with ECRH power and safety factor $q\left(I_{\mathrm{p}}\right)$ scanning. Given in Figs. 8 (a) and 8 (b) are the auto-power spectra of floating potentials, which are roughly proportional to radial electric field power spectra, considering that the range of radial wave vector of the potentials is very limited as shown in Fig. 6 (a). The intensities of the LFZFs and GAMs increase as the ECRH power increases from 380 to $680 \mathrm{~kW}$ while $q$ is fixed and the collision frequency is estimated to be $3 \times 10^{3} / \mathrm{s}, 2 \times 10^{3} / \mathrm{s}$, and $1 \times 10^{3} / \mathrm{s}$ for ECRH powers of 0,380 , and $680 \mathrm{~kW}$, respectively. Nevertheless, the power fractions of the LFZF, GAM, and AT are $0.02: 0.18: 0.80$ and $0.09: 0.14: 0.77$ for ECRH power of 380 and $680 \mathrm{~kW}$, respectively, showing increase of the ZF fraction with ECRH power, but it is still lower than the Ohmic case. The GAM frequency increases with the ECRH power due to increase of the plasma temperature. On the other hand, the intensity of the LFZF and GAM increases and decreases, respectively, as the edge safety factor decreases from 6.2 to 3.5. This is consistent with the theoretical prediction [17] and the observations in TJ-II stellarator, where the edge safety factor $q$ is low, so that the LFZF occurs in absence of the GAM [18].

\section{Summary}

In summary, the measurements with the three dimensional set of Langmuir probe arrays have unambiguously demonstrated the coexistence of intensive LFZFs, GAMs, LFFs and HFAT in the edge of HL-2A tokamak plasmas. The tempo-spatial characteristics of the floating potential fluctuations are analyzed in detail. The dependence of the intensities of the LFZFs and GAM on ECRH power and $q$ are studied. The radial wave number-frequency spectra of the LFZF show that the LFZF packets propagate outwards and inwards as equally likely events, while the GAM packets propagate predominantly outwards. The three wave coupling of the zonal flows, including the GAMs, and the LFFs with the HFAT is investigated.

The authors thank the HL-2A Team for operation of the machine. This work is supported by NSFC Grant Nos. 10775044 and 10675041, the Sichuan Youth Foundation of Science and Technology, Grant No 09ZQ026-079, the National Basic Research Programme of China under Grant Nos. 2008CB717806 and 2009GB105005, and the JSPSCAS Core-University Program.

[1] Z. Lin et al., Science 281, 1835 (1998).

[2] H. Biglari et al., Phys. Fluids B2, 1 (1990).

[3] P.H. Diamond et al., Plasma Phys. Cont. Fusion 47, R35 (2005).

[4] A. Hasegawa et al., Phys. Rev. Lett. 59, 1581 (1987).

[5] L. Chen et al., Phys. Plasmas 7, 3129 (2000).

[6] N. Winsor et al., Phys. Fluids 11, 2448 (1968).

[7] M. Jakubowski et al., Phys. Rev. Lett. 89, 265003 (2002).

[8] A. Fujisawa, Nucl. Fusion 49, 013001 (2009).

[9] K.J. Zhao et al., Phys. Rev. Lett. 96, 255004 (2006).

[10] T. Ido et al., Nucl. Fusion 46, 512 (2006).

[11] A. Fujisawa et al., Phys. Rev. Lett. 93, 165002 (2004).

[12] A.D. Liu et al., Phys. Rev. Lett. 103, 095002 (2009).

[13] T. Lan et al., Plasma Phys. Cont. Fusion 50, 045002 (2008).

[14] K.J. Zhao et al., Phys. Plasmas 14, 122301 (2007).

[15] K.J. Zhao et al., Nucl. Fusion 49, 085027 (2009).

[16] Y.C. Kim et al., IEEE Trans. Plasma Sci. 7, 120 (1979).

[17] N. Miyato et al., Phys. Plasmas 11, 5557 (2004).

[18] M.A. Pedrosa et al., Phys. Rev. Lett. 100, 215003 (2008). 